\title{
Reduction of SAR for bar type handsets with metal frame
}

\author{
Jae Hyun Choi ${ }^{1 \mathrm{a})}$ and Ju Wook Jang ${ }^{2 \mathrm{~b})}$ \\ ${ }^{1}$ Graduate School of Information and Technology, Sogang University \\ Seoul, Korea \\ ${ }^{2}$ Department of Electronic Engineering, Sogang University \\ Seoul, Korea \\ a)chaehyunni@sogang.ac.kr \\ b) jjang@sogang.ac.kr
}

\begin{abstract}
Handsets with metal frame, which are very popular recently, cause higher SAR value due to current leakage into the metal frame. We propose a new design to reduce the SAR (Specific Absorption Rate) without degrading antenna performance which is represented by TRP (Total Radiated Power). We place the contact points between PCB GND and the metal frame at the length of $\lambda / 2$. This distributes the current over wider area and hence reduces SAR. Besides, we extend the PCB GND around the antenna feed to reduce the high current density in the area. Our experiment shows $32 \%$ reduction in SAR without degrading TRP.
\end{abstract}

Keywords: SAR, TRP, Metal Frame, Handset

Classification: Science and engineering for electronics

\section{References}

[1] P. Halla, Specific Absorption Rate Design of 3rd Generation Handsets, Helsinki University of Technology, Finland, 2008.

[2] J. C. Lin, "Update of IEEE Radio Frequency Exposure Guidelines," $M i$ crow. Mag., vol. 7, no. 2, pp. 24-28, April 2006.

[3] International Commission on Non-Ionizing Radiation Protection (ICNIRP), "Guidelines for limiting exposure to time-varying electric, magnetic, and electromagnetic fields (up to $300 \mathrm{GHz}$ )," Health Physics, vol. 74, no. 4, pp. 494-522, April 1998.

[4] IEEE Std. C95.1 - 2005, IEEE Standard for Safety Levels with Respect to Human Exposure to Radio Frequency Electromagnetic Fields, $3 \mathrm{kHz}$ to $300 \mathrm{GHz}$, IEEE, New York, USA, p. 248, April 2006.

[5] N. Kuster and Q. Balzano, "Energy Absorption Mechanism by Biological bodies in The Near Field of Dipole Antennas Above $300 \mathrm{MHz}$," IEEE Trans. Veh. Technol., vol. 41, no. 1, pp. 17-23, Feb. 1992.

[6] S. I. Kwak, D. U. Sim, J. H. Kwon, and H. D. Choi, "Comparison of the SAR in the human head using the EBG structures applied to a mobile handset," Microw. Conf. European, pp. 937-940, 2007.

[7] S. I. Kwak, D. U. Sim, J. H. Kwon, and J. H. Yun, "SAR reduction of PIFA with EBG structures for mobile applications," IEIEC Trans. Commun., vol. E92-B, no. 11, Nov. 2009. 
[8] C. Z. Ning, Antennas for Portable Devices, John Wiley \& Sons, 2007.

[9] H. Nyberg and K. Jokela (Editors), Ionisoimaton säteily Sähkömagneettiset kentät (Non-ionizing radiation - Electromagnetic fields, in Finnish), Helsinki, Finland, p. 555, 2006.

[10] A. Cabedo, J. Anguera, C. Picher, M. Ribo, and C. Puente, "Multiband Handset Antenna Combining a PIFA, Slots, and Ground Plane Modes," IEEE Trans. Antennas Propag., vol. 57, no. 9, pp. 2526-2533, Sept. 2009.

\section{Introduction}

As mobile handsets are getting slim and full-touch displays are employed, metal frame is becoming a more popular choice to improve the handset chassis. But current tends to leak into metal frame, causing stronger electromagnetic field and human effects (head effect, hand effect). The specific absorption rate (SAR, $\mathrm{mW} / \mathrm{g}$ ) is a measure of such human effects. The SAR represents the radio frequency power absorption in human tissues [1]. Federal Communications Commission (FCC) requires that SAR value should be less than $1.6 \mathrm{~mW} / \mathrm{g}$ per $1 \mathrm{~g}[2,3,4]$. The SAR maximum in human tissue is usually found next to the location of maximum antenna current [5]. There has been a few previous schemes to reduce SAR [6, 7]. However, the schemes are either difficult to implement in compact sizes at operating bandwidth of mobile handsets or do not work with mobile handsets using metal frame. Usually increasing total radiated power (TRP) increases SAR in the user's head $[8,9]$. Thus it is challenging to improve TRP without increasing SAR. The maximum SAR of the high-band is known to occur due to currents concentrated in the shorting edge of the antenna $[8,10]$.

In this paper, we propose a new handset design which reduces SAR without degrading antenna performance (TRP). We place the contact points between PCB GND and the metal frame at the length of $\lambda / 2$ where $\lambda$ is the wavelength. This distributes the current over wider area and hence reduces SAR. We also tested other contact points which are related to resonance of radiation to ensure that design reduces $\mathrm{SAR}$ most without sacrificing TRP. Besides, we extend the PCB GND around the antenna feed to reduce the high current density in the area. Our experiment of the proposed design shows SAR of $1.61 \mathrm{~mW} / \mathrm{g}$ which is close to the FCC limit with TRP of $19.71 \mathrm{dBm}$. This is 32\% reduction in SAR and without degradation in TRP compared with a basic two-contact design shown in Figure 2 (a). The SAR of our design in Figure $3(\mathrm{~b})$ is $1.61 \mathrm{~mW} / \mathrm{g}$ which is close to the FCC limit with TRP of $19.71 \mathrm{dBm}$.

\section{Proposed scheme and experimental results}

First, we show how metal frame increases the SAR value and the present our design to reduce the SAR value. 


\subsection{Increase of SAR for bar type handset with metal frame}

We compare the distribution of magnetic field for the handset without metal frame and the handset with metal frame. For the handset with metal frame, magnetic field is more concentrated, resulting in higher SAR value which represents the maximum magnetic field in the human tissue [8]. This is due to the current leaked into the metal frame. The radiating current is coupled to the metal frame. And part of the current in metal frame flows in the same direction as in the PCB GND. Therefore, maximum magnetic field is increased and it increases the SAR. Besides the metal frame in handset causes the degradation of antenna performance in our measurement. TRP is reduced from $19.61 \mathrm{dBm}$ to $18.16 \mathrm{dBm}$ while $\mathrm{SAR}$ is increased from $1.33 \mathrm{~mW} / \mathrm{g}$ to $1.94 \mathrm{~mW} / \mathrm{g}$ with use of metal frame. Thus it is very challenging to meet TRP specification for antenna performance while reducing the SAR to the FCC limit of $1.6 \mathrm{~mW} / \mathrm{g}[2,3,4]$ when metal frame is used on the handset. To the best of our knowledge, our design is the first to achieve this goal. Previous designs with reduced SAR $[6,7]$ are either difficult to implement on the compact handsets of today due to the length requirement imposed by wavelength or not suitable for handsets with metal frame.

\subsection{Proposed design I (Place contact points at $\boldsymbol{\lambda} / \mathbf{2}$ length)}

Figure 1 shows the detailed dimension and VSWR (Voltage Standing Wave Ratio) of the basic design antenna structure in Figure 2 (a). The antenna in Figure 1 (a) has a feed terminal A connected to the feed element and a ground terminal B connected to the PCB GND. Signal is provided to the antenna using feed terminal $\mathrm{A}$ and ground terminal $\mathrm{B}$. The antenna has two paths for $900 \mathrm{MHz} / 2 \mathrm{GHz}$ operation and the feed element and the coupled parasitic element are not electrically connected. The feed element serves as an antenna at about $2 \mathrm{GHz}$ and is near-field coupled to coupled parasitic element. Through this near-field coupling, the coupled parasitic element can resonate at about $900 \mathrm{MHz}$ and can also generate a higher-order resonant mode at about $2 \mathrm{GHz}$. For achieving a resonant mode at about $2 \mathrm{GHz}$, the total length of the path $\mathrm{A}$ is chosen to be about a quarter-wavelength at $2 \mathrm{GHz}$. For achieving a resonant mode at about $900 \mathrm{MHz}$, the total length of the path $\mathrm{B}$ is chosen to be about a quarter-wavelength at $900 \mathrm{MHz}$.

To reduce the effect of the current on the metal frame, we tested several resonance points as possible contacts between PCB GND and the metal frame. Figure 2 shows three choices where $\lambda$ is the wavelength (set to $2 \mathrm{GHz}$ in our experiment). The design in Figure 2 (c) is chosen as Proposed Design I since it shows the minimum SAR with the highest TRP. The SAR of Figure 2 (a) is $2.38 \mathrm{~mW} / \mathrm{g}$ with $19.2 \mathrm{dBm}$, Figure $2(\mathrm{~b})$ is $2.41 \mathrm{~mW} / \mathrm{g}$ with $18.9 \mathrm{dBm}$ and Figure 2 (c) is $2.12 \mathrm{~mW} / \mathrm{g}$ with $19.6 \mathrm{dBm}$. We call the design in Figure 2 (a) as Basic Design and use it as a reference for our proposed designs. The reason for the minimum SAR for the chosen $\lambda / 2$ contact design can be explained by Figure 2 which shows the areas of radiation (circled area) for the three designs. Figure 2 (c) (Proposed Design I) shows the largest area of radiation. The largest area implies more distributed radiation, hence smaller 


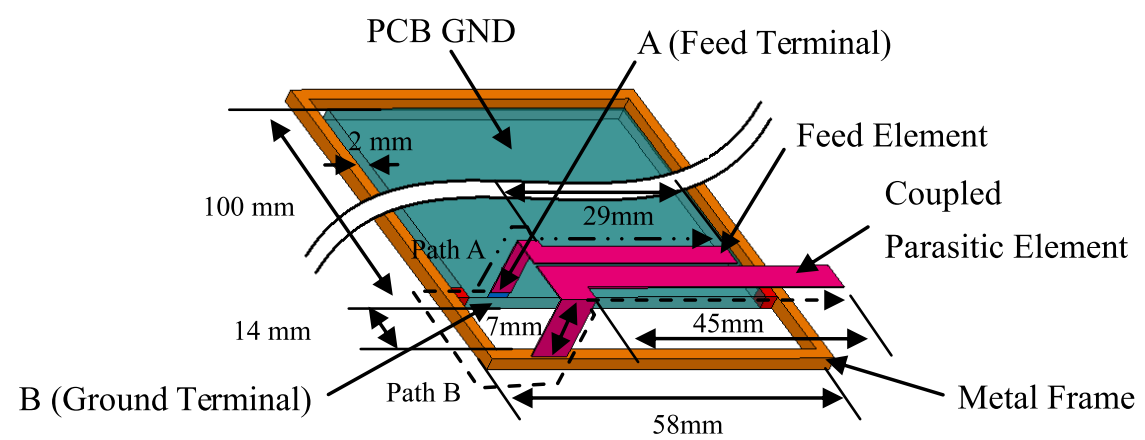

Path A $(-\cdots \longrightarrow)$ : feed element

Path B (- - - $\rightarrow$ ): coupled parasitic element

(a) Structure of basic design in Fig. 2(a)

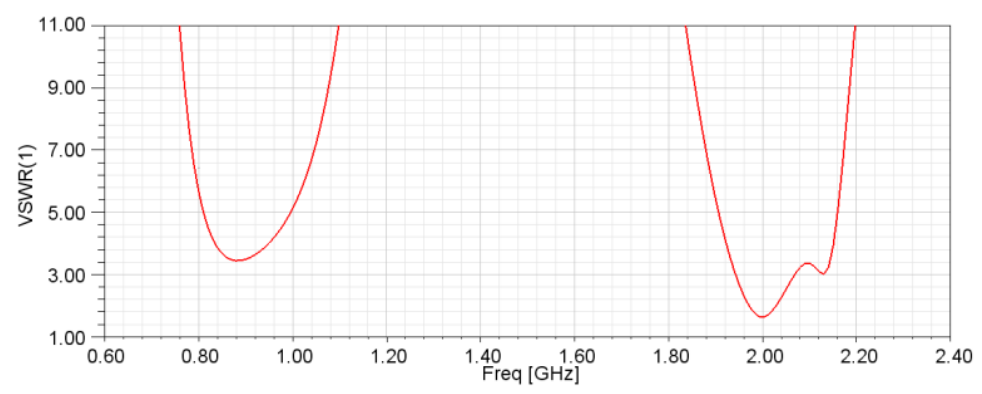

(b) VSWR(Voltage Standing Wave Ratio) of basic design in Fig. 2(a)

Fig. 1. Antenna mechanism of basic design in Fig. 2 (a)
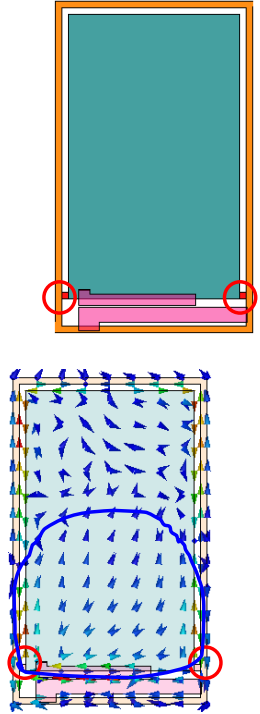

(a) Basic design
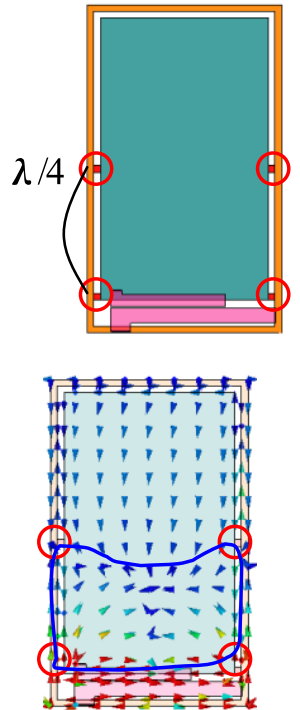

(b) $1 / 4 \lambda$ contact
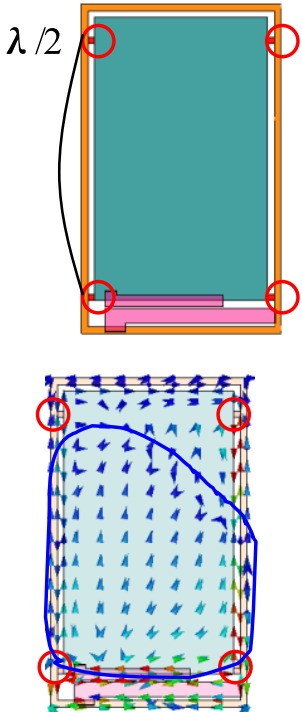

(c) Proposed design I

Fig. 2. Current distribution for handset designs with various contact points between PCB GND and metal frame 
SAR which represents the maximum magnetic field in the human tissue in the vicinity. We tested a few more resonance points. None performs better than our choice. As contact point moves to the vicinity of human head, the maximum point of current density also gets close to human head, leading to higher SAR. This explains the highest SAR value in which the contact points

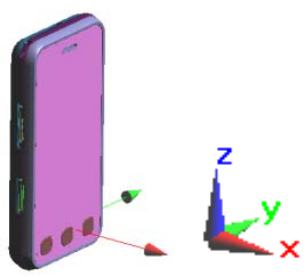

(X: Front(LCD)-human head direction, Y: Side, Z: Up)
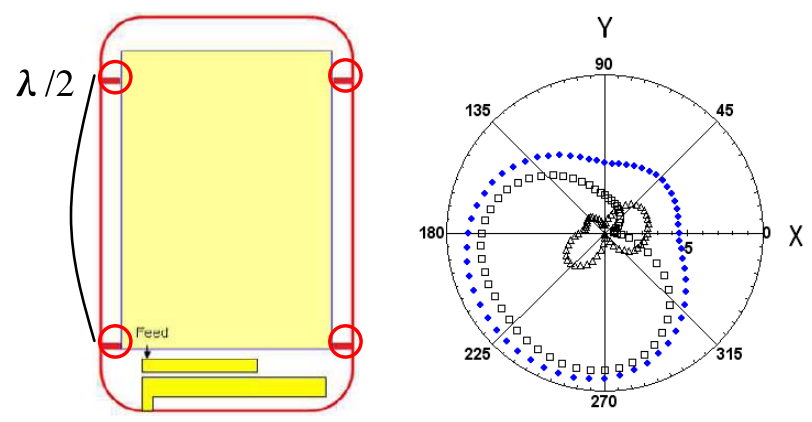

(a) Proposed design I
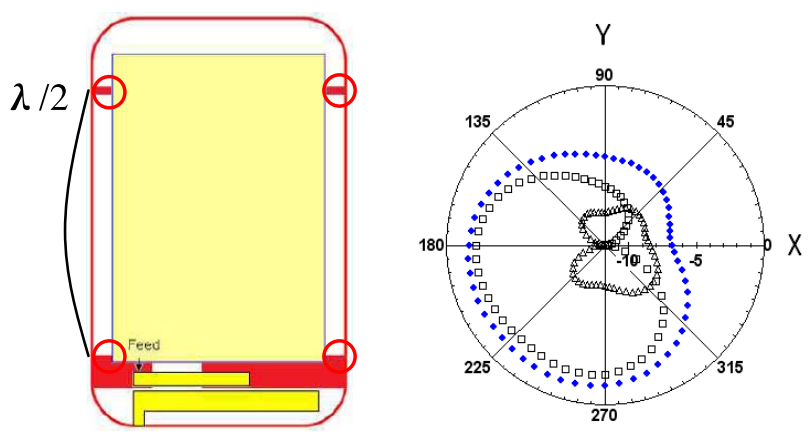

(b) Proposed design II

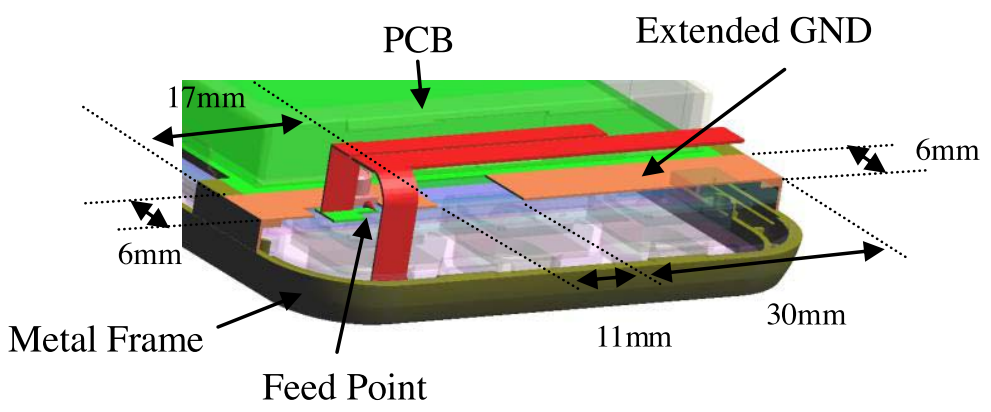

(c) Detailed structure of proposed design II

( : Total(Theta+Phi) directivity, $\square$ : Theta directivity, $\triangle$ : Phi directivity)

Fig. 3. Extension of PCB GND under antenna feed in 
are located in the middle which usually touches human head.

\subsection{Proposed design II (Proposed design I plus GND extension)}

While our proposed design I in Figure 2 (c) shows the SAR of $2.12 \mathrm{~mW} / \mathrm{g}$, it is still higher than the FCC limit of $1.6 \mathrm{~mW} / \mathrm{g}[2,3,4]$. To further reduce the SAR we extend the PCB GND to the area which is beneath the antenna feed. Figure 3 (c) shows detailed structure of the expanded GND plane. We call the resulting design as Proposed Design II which is our final design. This idea is based on our observation that the current density is very high near the antenna feed. Our scheme effectually positions GND at the bottom of the antenna pattern. Our test shows considerable reduction in SAR as shown in Table I. Now our design exhibits the SAR value of $1.61 \mathrm{~mW} / \mathrm{g}$ which is very close to the FCC restriction without degradation in TRP compared with the basic design shown in Figure 2 (a).

It implies that radiation toward head is reduced while retaining the same TRP. In Figure 3, X-axis represents the direction toward human head. The directivity of radiation is represented by blue dots. Note that the directivity in the direction of $\mathrm{X}$-axis is reduced in Proposed Design II compared with Proposed Design I.

Table I. SAR measurement for the designs

\begin{tabular}{|c|c|c|c|}
\hline & $\begin{array}{c}\text { Basic } \\
\text { design }\end{array}$ & $\begin{array}{c}\text { Proposed } \\
\text { design I }\end{array}$ & $\begin{array}{c}\text { Proposed } \\
\text { design II }\end{array}$ \\
\hline TRP & $19.2 \mathrm{dBm}$ & $19.6 \mathrm{dBm}$ & $19.71 \mathrm{dBm}$ \\
\hline SAR $(1 \mathrm{~g})$ & $2.38 \mathrm{~mW} / \mathrm{g}$ & $2.12 \mathrm{~mW} / \mathrm{g}$ & $1.61 \mathrm{~mW} / \mathrm{g}$ \\
\hline
\end{tabular}

\section{Conclusion}

This paper presents a solution to the problem of SAR increase due to use of metal frame for mobile handsets. First, we place the contact points between PCB GND and the metal frame at the length of $\lambda / 2$ where $\lambda$ is the wavelength. Second, we extend the PCB GND around the antenna feed to reduce the high current density in the area. Our experiment shows $32 \%$ reduction in SAR without degrading TRP.

\section{Acknowledgments}

This work was supported by the National Research Foundation of Korea (NRF) grant funded by the Korea government (MEST) (No. 2010-0014854). 